\title{
Stenting lohnt nicht
}

Fragestellung: Bietet Stenting von symptomatischen Vertebralisabgangsstenosen zur Schlaganfallprävention Vorteile?

Hintergrund: Vom Stenting bei symptomatischen Karotisstenosen ist bekannt, dass die Komplikationsraten etwas höher als bei der Operation, die Langzeitergebnisse aber vergleichbar sind. Für Vertebralisabgangsstenosen steht allerdings die Option einer Operation nicht zur Verfügung, sodass hier nur ein Stenting mit optimaler medikamentöser Therapie verglichen werden kann.

Patienten und Methodik: Die Studie schloss Patienten mit transienten ischämischen Attacken oder leichtem Schlaganfall und extrakraniellen (80\%) oder intrakraniellen Stenosen der Arteria vertebralis mit Lumeneinengung von über $50 \%$ ein, die entweder die bestmögliche medikamentöse Therapie oder eine medikamentöse Therapie in Kombination mit Stenting erhielten. Der primäre Endpunkt war vaskulärer Tod, Herzinfarkt und Schlaganfall bis 30 Tage nach Therapiebeginn.

Ergebnisse: Die Studie wurde nach der Randomisierung von 115 Patienten vorzeitig abgebrochen. 57 wurden gestentet und 58 allein medikamentös behandelt. In der Stentgruppe erreichten drei Patienten den primären Endpunkt, in der Medikationsgruppe einer (5\% vs. $2 \%$ ). Im Langzeitverlauf trat der kombinierte Endpunkt bei $19 \%$ der Patienten der Stenting- und

Compter A, van der Worp HB, Schonewille WJ et al; VAST investigators. Stenting versus medical treatment in patients with symptomatic vertebral artery stenosis: a randomised open-label phase 2 trial. Lancet Neurol 2015; 14: 606- 14

\section{Hier steht eine Anzeige.}

